\title{
EL TRÁNSITO DE LOS PROPÓSITOS A LOS EFECTOS EN LA EVALUACIÓN MASIVA DE LA EDUCACIÓN SUPERIOR ${ }^{1}$
}

\section{Resumen}

En el marco de la política de evaluación de la calidad de la educación, comienza en el país la aplicación de los exámenes de calidad de la educación superior -ECAES- orientados desde el concepto de competencias. Dicha estrategia reconoce su limitación para concluir, por si misma, acerca de la calidad de la educación superior. Por lo tanto, los usos posibles de los resultados no pueden ni abstraerse de tal consideración ni ser concluyentes para operar en contra o a favor de los sujetos e instituciones evaluados. Al desentrañar los sentidos de la evaluación masiva, para anticipar los posibles efectos de su uso, se reconoce que estos están en relación, más que con los propósitos desde los cuales se propone la prueba, con el instrumento y el modelo de análisis aplicado. La construcción del instrumento de prueba y del modelo de análisis reflejan la postura desde la cual se concibe el accionar educativo y evaluativo. De allí que problematizar sobre dichas posturas permita acercarse a la comprensión de los efectos de dichos supuestos.

Palabras clave: Evaluación, evaluación masiva, evaluación por competencias, exámenes de estado, calidad de la educación, evaluación de la educación superior.

\section{Abstract}

In the frame of evaluation policies on education quality, the application of quality tests to college education, -ECAES- based on the concept of competence, has already started in Colombia. Such a strategy recognizes its own limitation to draw conclusions about college education by itself. Therefore, the possible uses of its results must neither ignore the previous remark, nor be conclusive in operating against or in favor of individuals or institutions evaluated. After understanding the meanings of massive evaluation and, in order to anticipate possible effects of its application, it is acknowledged that these effects are related both to the instrument and the model of analysis applied, more that to the objectives prescribed for the test. Both the construction of the test as a tool, and

* Odontólogo. Magíster en Educación, Universidad Pedagógica Nacional. E-mail: gesoral@yahoo.com

" Odontóloga. Licenciada en Química. Magíster en Educación, Universidad Pedagógica Nacional. E-mail: sofijaco@hot mail. com

1 Este trabajo se desarrolló en el marco de la Maestría en Educaciòn de la Facultad de Educación de la Universidad Pedagógica Nacional, bajo la asesoría del profesor Guillermo Bustamante Zamudio. Fue recibido el 20 de octubre de 2003 y aprobado el 14 de noviembre de 2003. 
the model of analysis, show the stance adopted to conceive educative and evaluative actions. For that reason, to question such perspectives allows to get close to the understanding of the effects of those assumptions.

Key words: Evaluation, massive evaluation, evaluation on competence, State exams, education quality, college education evaluation.

\section{Consideraciones previas}

El Estado colombiano se ha propuesto evaluar la calidad de la educación superior. Esta decisión se inscribe en idéntico propósito para con los demás niveles educativos, actividad comenzada en nuestro país a comienzos de la última década del siglo XX. La política educativa se ha definido en función de la evaluación de la calidad y para la educación superior contempla los siguientes componentes: a) Exámenes de calidad de la educación superior (ECAES); b) Acreditación de programas e instituciones de educación superior y c) Estándares de calidad.

A propósito de estos componentes, se dice que ninguno se considera concluyente para hablar de la calidad de la educación superior, y que se busca coherencia entre todos los elementos de la estrategia de valoración de la calidad. Para el caso de las pruebas, en atención a que es la primera vez que se hacen y a que han sido elaboradas por equipos que no tienen tradición en evaluaciones masivas con el enfoque propuesto, es lógico presentar unos resultados sin olvidar el estado incipiente de desarrollo de los instrumentos utilizados.

Sin embargo, los antecedentes de acciones desarrolladas por los últimos gobiernos nacionales, específicamente en la educación básica, señalan en direcciones distintas: allí las evaluaciones han acaparado la atención y por sí mismas se consideran suficiente información para concluir sobre el estado de la calidad de la educación; además, los componentes propuestos para la evaluación se separaron y no hay solidaridad entre ellos. De otro lado, pese a que en la educación básica la situación frente a los instrumentos era muy parecida a la de los ECAES hace una década, nunca se habló de los resultados en función del estado de desarrollo del ejercicio evaluativo, sino como si dichos instrumentos ya fueran objetivos; es decir, no fue explícito que se trataba de unos primeros ensayos (el ICFES mismo decía a comienzos de los 90 que no sabía evaluar "calidad de la educación") y que, en consecuencia, los resultados hablarían más del estado de la investigación que de lo que los estudiantes realmente sabían.

En uno y otro contexto (educación básica y superior), la evaluación se propone, desde un ámbito político, no está puesta allí como una construcción disciplinar, no surge como propuesta de la educación (básica o superior) ni del sector educativo; se impone en consideración a otros requerimientos, tanto internos como externos al sector mismo. $Y$ no es que tenga que ser de otra manera, sino que tales iniciativas se han presentado como si provinieran exclusivamente de la educación.

Pero, sin siquiera poner en discusión la idea de la evaluación, ni la política con arreglo a la cual se ha dispuesto evaluar la calidad de la educación superior, es necesario tener en cuenta que hay diversas posturas desde las cuales problematizar un objeto de estudio. Esto implica que hay diferentes posiciones frente a la evaluación, frente al concepto de calidad, etc. Y cada una de estas posturas tiene sus referentes con- ceptuales, epistemológicos y lógicos específicos.

De manera que la academia debe preguntarse por esta manera de evaluar la educación superior, por su participación en ella, por la responsabilidad que le compete una vez los resultados comiencen a producir sus efectos, cada uno desde su contexto, pues cada profesión actúa en un marco histórico específico (por ejemplo, los de la salud se encuentran actualmente en un contexto de reforma sectorial, que obedece además a requerimientos que en muchos aspectos están por fuera del sector sanitario). Y además en un contexto social y un momento histórico en el que -según el [MEN, 2002]- una de cada cinco personas que terminan el bachillerato logran acceder a la formación universitaria, y sólo logran graduarse 3 de cada 1000 que ingresan. En ese contexto, las pruebas tendrán que proponer e interactuar con la realidad.

Hacia dónde se dirigen las pruebas, es una pregunta que los académicos de las diversas profesiones tienen que intentar responder, pues las consecuencias que se derivan de su aplicación y uso están estrechamente relacionadas con el diseño y el modelo de análisis de las mismas.

\section{Carácter aproximativo de los ECAES}

No solamente los ECAES por sí solos son insuficientes para emitir un juicio para valorar la calidad de la educación superior; también para 
valorar lo que le es específico, los aprendizajes de los estudiantes, el instrumento evaluativo supone unas limitaciones, relativas a todo instrumento, que es necesario reconocer para concluir en coherencia con las mismas.

A continuación, se enuncian cuatro tipos de argumentos para su consideración previa a la emisión de juicios, que exponen el carácter aproximativo de la prueba.

\subsection{Desde la naturaleza del objeto}

Si la prueba pretende acercarse a los saberes de los estudiantes, luego de un proceso formativo, entonces el objeto de la evaluación son los aprendizajes. Pero en la educación superior esto tiene una particularidad que es necesario destacar. Si bien allí se dan efectivamente aprendizajes, también es cierto que se enfatiza en el desarrollo de la esfera procedimental de los saberes; es decir, aprendizajes de tipo práctico para la solución de problemas. Sin embargo, los ECAES pretenden evaluar los aprendizajes de los estudiantes de la educación superior, en su mayoría con pruebas "de lápiz y papel" (en atención a los costos, entre otros), con las cuales difícilmente puede valorarse el saber procedimental. No quiere decir esto que los aprendizajes no procedimentales sí puedan ser observados y manipulados como un hecho empírico, pues son igualmente construcciones que hacen los sujetos en contextos particulares, procesos complejos y multideterminados en los que confluyen factores de diversa naturaleza (biológicos, psicológicos, socio-antropológicos, etc.). Quiere decir que la diferencia no se expresa en la forma única de evaluar.

De tal manera, si en la educación básica los aprendizajes se han sometido a una "medición" que descuida estas propiedades, en la educación superior el error se duplica, pues, de un lado, se toma el primer tipo de aprendizaje como un objeto medible y, de otro lado, los aprendizajes en la modalidad procedimental no se valoran mediante una observación sistemática de las acciones mismas a lo largo de un tiempo suficiente.

Entonces, a lo sumo, los ECAES podrán acercarse a los saberes declarativos de tipo comprensivo, donde se hacen explícitos unos enunciados de los qué, para qué y los cómo que subyacen como saberes declarativos a los procedimientos mismos. Poco se podrá averiguar sobre los saberes procedimentales, pues no existe una relación lineal entre la apropiación de un saber declarativo y de un saber procedimental, ni viceversa. Así como hay distancias entre el saber y la acción práctica, una ejecución técnica brillante no siempre está precedida de una sólida fundamentación teórica

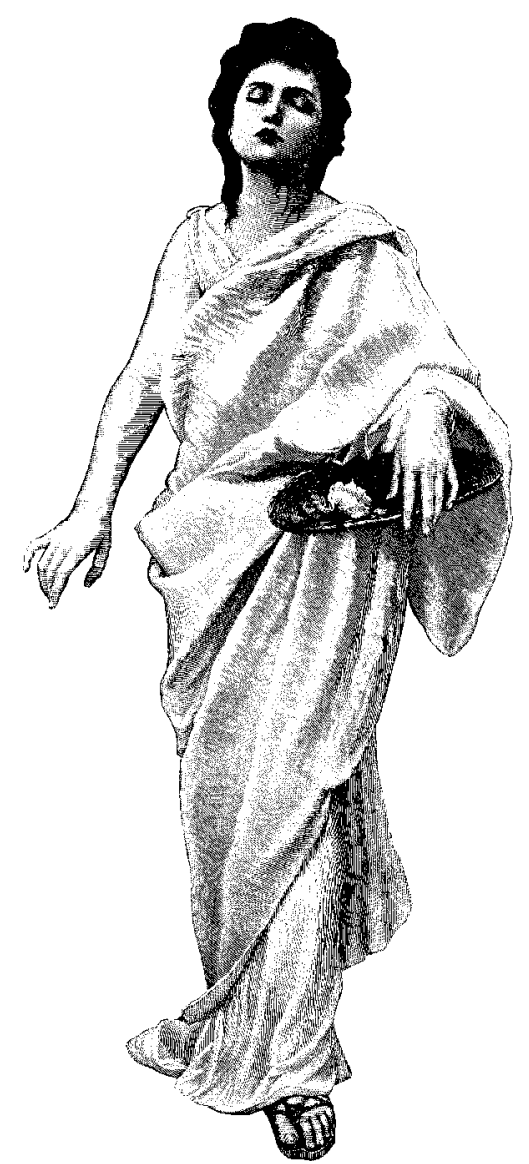

(aspecto fácilmente demostrable en la práctica odontológica). Avanzar en la valoración de los saberes declarativos en una aproximación comprensiva, más allá de lo memorístico, es un logro; sin embargo, no podría con ello asegurarse que se valoran los aprendizajes propiciados por la educación superior, en tanto éstos, para una gran proporción de disciplinas y saberes, enfatizan en los saberes procedimentales.

De otro lado, tal manera de evaluar subvalora la mediación de la relación afectiva que se establezca con el saber y con sus agentes facilitadores, asunto fundamental para entender la apropiación de cualquier saber, según los planteamientos contemporáneos de la psicología educativa y cognitiva [Ausubel, Novack \& Hanesian, 1983: 347-375]; [ Zubiría, M. 2002].

También debe referenciarse la naturaleza específica del objeto de estudio y trabajo en cada profesión. La variación de las características de una a otra, determina diferentes modalidades de apropiación, enunciación y exposición de un saber. Para las profesiones del campo de trabajo de la salud, por ejemplo, el objeto de estudio y trabajo es el proceso salud/enfermedad, que es una expresión individual y colectiva multidimensional, donde opera la variabilidad biosicosocial. No se trata, entonces, de un objeto similar al de las ciencias exactas ni físicas.

Frente al objeto de estudio y trabajo de la práctica odontológica, el proceso salud-enfermedad [Breilh, J. 1994. Citado en: Nieva, BC. Jácome, S. Cendales, L. 1998] expone lo siguiente:

Los estudios económicos, demográficos, históricos y sociológicos han confirmado el hecho de que el comportamiento de los procesos humanos de adaptación, llamados enfermedades por nosotros, han cambiado a través de la historia... así mismo, la antropología ha mostrado que la respuesta de los individuos ante un mismo proceso 
de cambio no es siempre la misma y que existen elementos culturales que influyen en la percepción de los síntomas y en el comportamiento frente a ellos. Es decir, que "el contexto sociocultural interactúa con lo genético, lo biológico y lo psíquico en el proceso de organización de las formas mismas de presentación, representación y modificación de los procesos vitales del individuo.

La responsabilidad profesional se orienta hacia la constitución de un conjunto de saberes y prácticas que ofrezcan soluciones a este proceso de variabilidad -alternativas para una realidad compleja y heterogénea que no son únicas y excluyentes-. Una prueba como los ECAES permite explorar unas posibilidades de respuesta restringidas a los condicionamientos que otorga el instrumento; una prueba "de papel y lápiz", de selección múltiple y única respuesta, con un tiempo definido para su resolución, aún en los mejores intentos, es un pobre reflejo de los contextos de acción profesional en la resolución de problemas. Estos contextos, más bien, están marcados por múltiples posibilidades, opciones complementarias, todas con campos de validez relativos, de acuerdo con las condiciones, necesidades y requerimientos del contexto y de los sujetos; allí los tiempos son flexibles y los recursos para acompañar la decisión de las opciones tomadas son múltiples. El ejercicio de simulación de situaciones problemáticas en la prueba resulta interesante, pero absolutamente insuficiente para dar cuenta de lo complejo que resulta la acción profesional en la práctica.

\subsection{Desde el concepto de competencia}

Las competencias (objeto de evaluación), se pueden entender de dos formas [Torrado, 1999: 30]:

- Como conocimiento abstracto, universal e idealizador; algo que está en el ámbito de lo esencial y alude a teorías sobre capacidades abstractas e innatistas de la mente, y que debería ser expresado por el individuo en las pruebas, independientemente de las situaciones a las que se enfrente.

- Una segunda forma, de mayor adopción en nuestro país, asume las competencias como una capacidad de realización situada y afectada por el contexto en el cual se desenvuelve el sujeto y la actuación misma. En ese caso, las competencias se piensan en consideración con los contextos que las hacen posibles, mediados por lo cultural, el lenguaje y los modos de hacer. Según Marín [2003: 101] esto no alude a una capacidad preformada o preconstituida, sino a un despliegue y creación de sus posibilidades dadas por la provocación de situaciones específicas.

Para cualquiera de las dos acepciones no se puede pretender que el desempeño, la realización, la conducta o la acción que se evidencia -como las respuestas en una prueba-, corresponden a la condición llamada "competencia" (o capacidad, inteligencia, potencialidad, etc.). En este sentido, diferentes disciplinas, como la filosofía, la psicología y la sociología han manifestado que no existe tal relación simétrica e isomórfica, que en términos del examen, equivaldría a relacionar los resultados de una prueba única de lápiz y papel con la posesión de una competencia determinada, propiciada ésta por procesos formativos intencionados por la educación superior; tal posición encuentra arraigo en posturas tradicionales y hegemónicas en el dominio de la ciencia y la producción de conocimientos, que caracteriza Díaz [1996: 176] de la siguiente forma:

"El paradigma dominante de la evaluación escolar parte de la ideología positivista de la generalización empírica verificable del aprendizaje de un saber, o de una actuación segmentada (destreza, habilidad, etc.). El saber o la actuación segmentada se asumen como aprendizajes uniformemente asimilados... en este sentido, la evaluación verifica o confirma el aprendizaje. La generalización (saber-no saber) se basa en la recolección u observación de los hechos (el comportamiento observable de los sujetos evaluados)".

\subsection{Desde el concepto de contexto}

Hecha la claridad anterior, asumimos, como lo expresa Díaz, [1996: 182], que "los procesos de adquisición o aprendizaje del conocimiento están histórica, social, cultural y contextualmente determinados"; 0 , como lo menciona Marín [2003: 107], que "aunque se dice explícitamente que las competencias son capacidades individuales, es indudable que dichas capacidades se generan o tienen sus condiciones de posibilidad dados unos marcos históricos, socioculturales determinados". Si es así, tendríamos que concluir que es muy limitada la capacidad de los exámenes para indagar por el contexto en el que se produce el aprendizaje; y, de otro lado, que es necesario interpretar dicho contexto en relación con el país y con la educación que tenemos. La forma como se entienda la noción de contexto será definitiva para la referencia analítica que se pueda obtener con los resultados de la prueba. Entender el contexto de una forma o de otra, orienta la interpretación de los resultados en ese mismo sentido.

Al hacer referencia a las nociones de contexto asumidas por las experiencias de evaluación de competencias en Colombia, Marín -en el texto en mención- encuentra una amplia diversidad de conceptos: por contexto puede entenderse la escuela, la cotidianidad, la disciplina, el entorno cultural, las situaciones nuevas o predeterminadas y la situación del examen, principalmente.

Por otra parte, algunas de las conclusiones del proceso de "evaluación de la calidad" en educación básica [DNP, 1994] sostienen que una se- 
rie de factores propios de la institución escolar, tales como las características del plantel, los procesos pedagógicos, la cualificación de la planta docente, la dotación del plantel, la disponibilidad de textos, entre otros, están asociados a un porcentaje del rendimiento de los estudiantes, situado entre el $25 \%$ y el $30 \%$. Esto ha sido denominado efecto colegio. Es decir, por más que la institución disponga de la mejor estructura, infraestructura y organización, puede explicar hasta un $30 \%$ del aprendizaje; el otro $70 \%$ está por fuera de la escuela, y consiste en la historia del estudiante, las condiciones que le han permitido llegar a conformarse como sujeto de determinada forma, relacionado con la estructura social, económica y el entorno cultural en el cual se está inmerso. En este sentido, Barrantes [2003: 164] ratifica que

El efecto o fenómeno colegio, retomado de la Organización para la Cooperación y el Desarrollo Económico [OCDE], consiste en precisar la importancia relativa del colegio (como un todo) en la explicación de la variabilidad en el desempeño de los alumnos (SED/DNP, 1999), y que para el Distrito Capital en la relación de factores con los resultados de una prueba, (SED/DNP 1999) el 24,6\% de la variabilidad del logro es explicada por las características de los planteles, el restante porcentaje $75,4 \%$ es desconocido, aún cuando supone una proporción mayor en la determinación de los logros y la calidad educativa.

Con base en esto, planteamos que los desempeños de los estudiantes no pueden ser tomados como una evidencia que opere en contra o a favor del estudiante -en tanto la prueba es individual-, o en contra o a favor de la institución -en tanto se supone que los resultados de los estudiantes se deben a la formación dispensada por ella-. Entonces, con base en estos resultados sólo se puede afirmar algo que ponga en relación unas condiciones individuales de los sujetos evaluados, con unas condiciones institucionales; $y$ ambos, en relación con unas condiciones sociales, económicas, po- líticas y culturales, que les subyacen y los determinan. Por lo tanto, si con base en los resultados de los ECAES se toman acciones en contra o a favor de los sujetos o de las instituciones, sería tornar responsable a los sujetos y a las instituciones, por asuntos que existen y los determinan, más allá de sus capacidades. De allí que resulte definitiva la noción de contexto que se asuma en la concepción de la prueba y desde la cual se analizan los resultados.

\subsection{Desde la idea de prueba psicométrica}

Una última consideración referida a lo que exponen los estudios psicológicos respecto a la valoración de las capacidades o inteligencias. Estos estudios sostienen que el desarrollo de las capacidades depende de unas condiciones del sujeto, de tipo biológico, genético y fisiológico, pero también de unas posibilidades socioculturales, en donde el dispositivo escolar es un referente importante. De otra parte, cuando un sujeto se enfrenta a una prueba, también entran en juego diversos aspectos relativos a la prueba y a logística misma, asuntos que se alejan de las condiciones que en la cotidianidad, que en la práctica, afronta el sujeto, razón por la cual se requieren de diversas modalidades de observación y registro, en una combinación de instrumentos, tiempos y actividades para acercarse a una valoración de las capacidades; información construida que, de todas formas, se encuentra en relación con los instrumentos y fundamentos teóricos utilizados.

Desde que se comenzaron a aplicar test para valorar las capacidades mentales, hubo controversia. Por ejemplo, a mediados del siglo XX, Wolff [1947: 225-227] afirma que en las pruebas están en juego no sólo las lógicas desde las cuales fueron construidos los test -que los análisis sociológicos y antropológicos se han encargado de cuestionar-, sino que allí también operan, como condicionantes de los resultados, el tiempo de la prueba, las aptitudes de los evaluados para relacionarse con estas, el ambiente de la prueba, incluso la forma y el orden como se presentan los ítems. Por su parte, las teorías de las inteligencias múltiples han cuestionado aquellas pruebas que persisten sólo en el acercamiento al nivel cognitivo de las capacidades, asunto que sobredimensiona el aspecto psicológico de las capacidades mentales, simplificando la complejidad que supone la determinación de dichas capacidades, desconociendo a su paso otros factores igualmente importantes, como los de tipo biológico y socioantropológico e incluso otros niveles de la dimensión psicológica como el afectivo. Al respecto, Zubiría [2002] plantea:

Pero entre todas una sobresale, sobresale entre demasiadas otras razones porque la desterró y la envió al ostracismo el siglo de la "inteligencia”. Siglo arrodillado que reverenció la lógica racionalista fría, finalizante con la moribunda psicología cognitiva. Siglo que pensó al ser humano como manojo de pensamientos, razonamientos y computaciones. $Y$ aunque la psicología cognitiva representó un paso adelante contra la visión mecanicista promovida por el conductismo, no bien, ella misma comienza a ser superada por una nueva interdisciplina, la neurociencia encargada de develar la mente.

En Pedagogía Conceptual el sistema afectivo define una dimensión fundamental de la mente humana, que, curiosamente, recién se descubre. El sistema afectivo aporta las motivaciones, las actitudes, los intereses, las expectativas que echan a andar, orientan, dirigen y sostienen al Sistema Cognitivo. ¿Hay algo con mayor valor que comprender el motor mismo de la mente? El sistema afectivo regula los aspectos vitales y significativos existenciales de todos los individuos.

Adicional a poner las motivaciones, las actitudes, los intereses, las expectativas, el sistema afectivo cognitivo orienta las relaciones con los otros. Y las relaciones satisfacto- 
rias con los otros son definitivas... Subjetividades difusas y dispersas, dotadas con variadas edades, tamaños, jerarquías, talentos, preparación, etc. Subjetividades con creencias, sentimientos, que desempeñan numerosos roles, las más contradictorios. Subjetividades u "objetos dotados con mente" complejísimos de descifrar e inteligir... el sistema afectivo permea la vida de todos los seres humanos y prácticamente en todo momento. Todo desempeño social meritorio (importando poco si es científico o tecnológico o artístico) involucra al Sistema Afectivo. Trátese de formular una teoría electrónica original o de presentar una sinfonía o mercadear un programa para computador o elegir a la mejor mujer para construir una familia o armar un equipo de fútbol o dirigir un colegio con metodologías innovadoras. Cualquier actividad humana significativa pasa por la mente afectivointerpersonal, la atraviesa de principio a fin.

Entonces, en atención a la posibilidad de valorar sólo algunas dimensiones del potencial de los estudiantes, se deben explicitar los alcances de las pruebas, de forma tal que no se conviertan en un elemento de segregación, en primera instancia, al sobredimensionar aspectos que están potencialmente mejor desarrollados y distribuidos en algunos sujetos, dadas unas posibilidades de acceso y utilización de unas herramientas culturales; $y$, en segunda instancia, al olvidar el papel que juegan, en la apropiación de un saber, otras esferas que pueden estar tanto en los mismos sujetos como más allá de ellos.

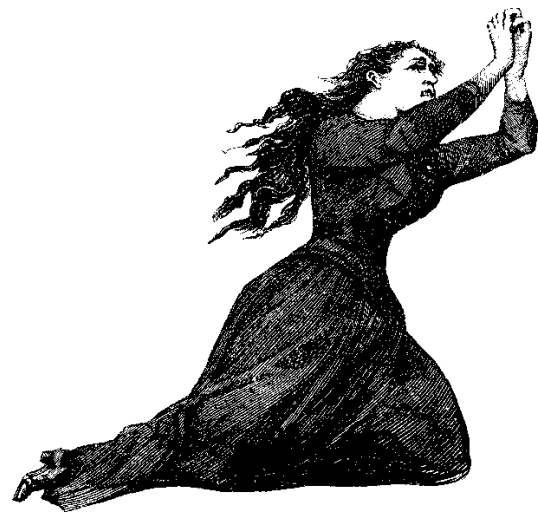

\section{El contexto, según los informes oficiales}

Luego de mencionar las consideraciones que indican el carácter aproximativo de la prueba y antes de definir qué tipo de análisis e interpretación podrían tener los resultados, presentamos algunos datos en donde se expone el contexto de la educación superior.

\subsection{Una "reunión técnica"}

En enero de 2002, en la reunión técnica denominada "El futuro de la Educación Superior en Colombia", el DNP plantea dos alternativas para ingresar en la educación superior: la universidad pública y la privada. El valor promedio de la matrícula en instituciones oficiales se encuentra entre $\$ 154.500\left(1 / 2 \mathrm{smlv}^{2}\right)$ y $\$ 1.081 .500$ (3.5 smlv) por semestre, mientras que en las instituciones de carácter privado el valor promedio es de $\$ 1.520 .000$ (5 smlv). Así mismo, plantea que, a pesar de la diferencia de costos, los estudiantes se han movido hacia la universidad privada, la cual agrupa el $74 \%$ de la nueva demanda ocurrida entre 1990 y 1999. No se habla de la composición social de ese movimiento; es como si todos los aspirantes a la universidad, independientemente de su extracción social, "se movieran hacia la universidad privada", con lo que queda implícita una idea de "preferencia", debida con seguridad a la "calidad" que encuentran allá (esto se confirmará más adelante, cuando sea el turno del MEN).

Además, el DNP plantea que la disponibilidad de ingresos es la mayor limitante para acceder al sistema educativo, y ahí sí presenta los datos de la última encuesta nacional de hogares para evidenciar la desigualdad de ingresos y por tanto de posibilidades. Es la idea que va a justificar hablar luego de que cuando los aportes se hacen a la universidad, hay una distribución

\footnotetext{
2 Salario mínimo legal vigente a la fecha.
}

indiscriminada que resulta inequitativa.

Respecto a la oferta pública, menciona que los recursos del Presupuesto Nacional que se entregan anualmente a las universidades ascienden a cerca de $\$ 1.1$ billones; de tal manera, el costo por estudiante en una institución pública es aproximadamente de 5.2 millones anuales. Obsérvese que hacen visibles las cifras que evidenciarían que al Estado le convendría más entregar ese promedio a cada estudiante (que con ese monto podría estudiar en una universidad privada, de mejor calidad, según queda insinuado), que sostener las universidades públicas.

Informa también el DNP que la ampliación de la cobertura implicaría aumentar la infraestructura y dotación de algunas instituciones, lo cual aumentaría el costo por estudiante en cerca del $60 \%$. De nuevo, esto deja un implícito, sobre todo con el trasfondo del ajuste fiscal: las universidades públicas serían un desperdicio de dinero, la universidad privada, "preferida" por la mayoría, haría la inversión respectiva, con cargo a su propio presupuesto; con lo que el gobierno gana en ampliación de la cobertura y no tiene que invertir.

En la misma reunión, el MEN considera que la educación es de calidad cuando se alcanzan los objetivos propuestos. Cosa que no ocurre en nuestro caso, pues, de un lado, la cobertura es apenas del 13 $\%$, estimada en la población entre 17 y 24 años; y, de otro lado, de cada 1000 estudiantes que ingresan a la universidad, sólo 3 logran el título. Además, los "objetivos propuestos" de los que habla ya se comienzan a poner en términos de los que alcanzan los estudiantes, cosa que no puede apuntar más que a resultados obtenidos en pruebas; es la justificación de los ECAES como condición necesaria para saber sobre la calidad, la cual ya está mal parada ante las cifras de cobertura 
y graduación. De ahí que "reconozca" que hoy no se mide el resultado del proceso educativo y que el Estado ejerce una discontinua vigilancia del sistema, más bien a partir de las quejas recibidas.

EI MEN plantea que "una educación de mala calidad es un engaño social, económico y político"... y se supone que todos debemos oponernos al engaño, sea mediante las medidas que sea. Por eso menciona una oferta desigual en términos de calidad, al pie de las siguientes cifras: el sistema de educación superior cuenta con 264 instituciones, de las cuales el $70 \%$ son privadas y el $30 \%$ son públicas; éstas últimas al borde de la quiebra (afortunadamente no son la mayoría). Es necesario, entonces, velar por la calidad, ante las deficiencias en el sistema de educación superior, que producen una confusión en la ciudadanía. Agrega que existen restricciones en el acceso debido a oportunidades limitadas en el crédito. En consecuencia, y para contrarrestar la inequidad señalada más atrás, es más equitativo dar los aportes a los aspirantes (por ejemplo, mediante becas o préstamos condonables), con lo que la universidad tendrá que resultar atractiva para tales aspirantes, a riesgo de desaparecer; y la manera más expedita es mediante los resultados en los ECAES.

Seguidamente afirma la condición de atomización de entidades, programas y títulos de las más variadas calidades y tamaños, de desiguales dotaciones e infraestructura disímiles; afirma que el suministro de información a la sociedad no es transparente ni veraz, que se basa en conceptos vendidos sin contenido real, tales como "calidad", "investigación", "excelencia" que, al manejarse irresponsablemente posibilitan la incertidumbre y la captura engañosa de un mercado en donde la expansión de la cobertura y la rentabilidad es inversamente proporcional a la calidad. No se dice, claro está, que la gran mayoría de esas instituciones funcionan con autori- zación oficial y que si ha habido una proliferación indiscriminada de oferta universitaria de mala calidad, ha sido por una apertura en las reglamentaciones, bajo la idea de que el mercado controlaría el asunto.

Finalmente concluye que el acceso a la educación superior, si no va unido a la calidad, no garantizará la equidad y superará la exclusión existente. Y que la evidente diferenciación de las instituciones y los programas por su calidad dará lugar a la inequitativa formación de profesionales con títulos de primera y de segunda, y hasta de tercera, convirtiéndose así en un factor generador de exclusión en la medida en que la baja calidad los discrimina y los excluye, por lo menos, de la posibilidad de participar activamente en la vida social y competir en igualdad de condiciones por un cupo en el mercado laboral.

\subsection{Propósitos del análisis de los resultados}

Se parte de la idea que el análisis de los resultados concreta los propósitos y explicita los usos posibles de una información obtenida por unas pruebas de las que se reconoce una serie de limitaciones. El uso de los resultados no necesariamente depende de los propósitos que se enuncian a la hora de justificar implícita o explícitamente los ECAES, ni de los propósitos desde los cuales las profesiones creen hacerse cargo de las pruebas. El uso de los resultados parece manifestarse más en la instrumentación de la prueba, expresada en su diseño y en el modelo de análisis. Por lo tanto, la responsabilidad por sus usos y, en últimas, por sus efectos, no puede abstraerse de quienes proponen y diseñan la prueba.

Retomando lo planteado por el DNP y el MEN, podría preverse que los resultados de la prueba van a ser utilizados para legitimar acciones sobre los sujetos y, sobre todo, sobre las instituciones mal ubicadas en la evaluación, a nombre de la "ca-

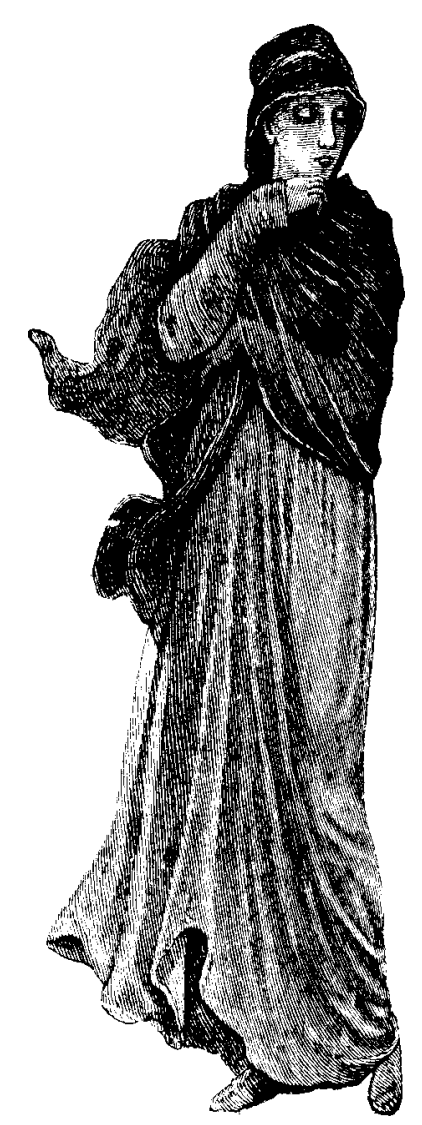

lidad" de la educación superior. No obstante, antes de aplicar una prueba existen elementos de juicio que dan cuenta de la heterogeneidad estructural del sector, en donde las posibilidades de acceso están en relación directa y preponderante con la capacidad adquisitiva.

Ya este uso ha sido advertido recientemente por el Consejo Nacional De Rectores [ASCUN, 2003]:

Se ha venido sosteniendo que los ECAES servirán de instrumento, aunque no es el único, para evaluar y medir la calidad de las IES. Reconocemos que estos exámenes pueden permitir una aproximación a los logros alcanzados en el proceso formativo de unos estudiantes, de una cohorte, de un programa académico y que esta información puede ser valiosa e importante, pero como lo dice la Asociación Colombiana de facultades de ingeniería-ACOFI-en una reciente declaración pública: «los resultados de 
estos exámenes no pueden ser interpretados por fuera del contexto del Sistema de Aseguramiento de la Calidad de la Educación Superior en nuestro país $y$, por lo tanto, no permiten emitir un juicio absoluto sobre la calidad de un determinado programa. Tampoco se espera que se reduzca a una lista de puntajes para establecer un escalafón de programas porque la calidad es plural, los valores que cada uno legítimamente quiere promover son diversos y sus características son diferentes. De este examen no deben derivarse calificativos excluyentes ni estigmatizaciones de ninguna especie. No se trata de una competencia individualista de programas sino de una sana emulación para que cada uno se supere a sí mismo» (ACOFI, septiembre de 2003: Declaración pública sobre los exámenes de calidad de la Educación Superior, ECAES en Ingeniería, 2003, punto 5).

De esta forma, una utilización punitiva de los resultados ocultaría el hecho de que el acceso a determinadas instituciones no está en relación con las capacidades individuales, sino con las posibilidades materiales. De ser utilizados los resultados de los exámenes como mecanismo de selección, se convertirían en un mecanismo más de exclusión y una total contradicción con los propósitos desde los cuales se proponen y diseñan los exámenes, y con el hecho de que todas las instituciones previamente han sido aprobadas por el ente rector del sector educativo para ejercer su función y ofrecer programas de formación profesional; sería simplemente una legitimación del estado actual de las cosas y una intervención a favor de su profundización.

\section{Algunas lógicas de análisis de resultados}

Al reconocer la heterogeneidad en el sector, tanto de sujetos como de instituciones, el carácter complejo y multideterminado del objeto de la evaluación, también tendría que hacerse la pregunta respecto a la lógica de análisis de los resultados de la prueba, la pregunta por cómo ope-

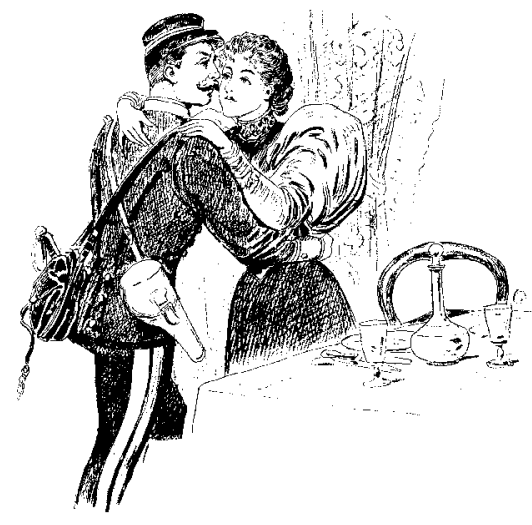

ran dichos modelos, cuáles son los supuestos bajo los cuales mantienen su rigor metodológico.

Ubicamos entonces que el análisis estadístico ha sido y es la herramienta analítica de mayor uso, no sólo en cierto tipo de investigación empírica, sino también en la evaluación psicométrica que opera bajo similares concepciones. Frente al uso de dicha herramienta, Páramo [1999:32] menciona que las restricciones de un análisis bajo un modelo estadístico lineal convencional suponen que los objetos que hacen parte de este análisis, requieren de un número similar de variables, requieren que la disposición de la información sea idéntica, requieren de regularidades. Y que debido a la eficiencia de los computadores y la elegancia matemática, los modelos estadísticos se han restringido para aquellos datos que están basados en el principio de tener dimensiones lineales, y que consecuentemente generan modelos explicativos dimensionales.

Por su parte, Simiand [1988:232, 234], al entender el método estadístico como una forma del método experimental, afirma:

La primera precaución que debemos tomar para no engañar a otros, ni engañarnos a nosotros mismos con nuestras abstracciones estadísticas consiste en preocuparnos porque nuestras expresiones de hechos complejos, nuestras medias, nuestros índi- ces, nuestros coeficientes, no sean resultados de cómputos cualesquiera, de combinaciones arbitrarias entre cifras y cifras, sino que modelen a partir de la complejidad concreta, respeten las articulaciones de lo real... para que tenga alguna correspondencia con la realidad, la primera condición es que nuestras expresiones estadísticas estén establecidas sobre una base que presente cierta homogeneidad, o también sobre una base que tenga una extensión apropiada, una extensión oportuna.

Finalmente, se pregunta: “ ¿no se les debe utilizar en complemento recíproco o en un cotejo útil, para poner de relieve los límites de su valor y de su legítimo empleo?". Pues bien, al revisar los métodos utilizados para la evaluación en educación, se encontró el modelo de Rasch como uno de los que más se publicita en este momento. Dicho modelo se define [Ceneval, 1999:3] como

\begin{abstract}
Un modelo que establece la probabilidad de respuesta de una persona ante un estímulo dado, en términos de la diferencia entre la medida del rasgo de la persona (lo medido) y la medida del estímulo utilizado. El modelo establece que la medida del rasgo de la persona es independiente del conjunto de estímulos aplicados. Así mismo, se dice que la medida de cada estímulo es independiente del conjunto de personas a las que se le somete. En rigor es la diferencia de medida de rasgo y medida de estímulo la que es independiente del instrumento o de la población. El modelo requiere que la variable sea unidimensional, ordenada e inclusiva.
\end{abstract}

Consideraciones que, contrastadas con los elementos expuestos hasta aquí, no serían las más adecuadas para el acercamiento a un fenómeno complejo como lo es el aprendizaje. Otros investigadores [Bustamante y Díaz, 2003:224-225], al exponer la manera como se utiliza la estadística para la evaluación de la calidad en Colombia, identifican por lo menos dos tipos de problemas: a) que los criterios técnicos condicionan en gran parte los criterios teóricos, pedagógicos y educativos (de donde 
la educación cede su especificidad a aquella de la estadística)... el hecho de reducirla a los elementos más asequibles, al margen de su relevancia. $Y$ b) que las lógicas internas de los modelos estadísticos se violentan para ajustar los resultados a lo deseado: selección y descarte arbitrario de variables, asunción de correlaciones de variables que no son de la misma naturaleza, operación con puntajes que no son equiparables (dado que no son parte de un mismo objeto ni comparten la escala de análisis particular), y la asignación de causalidades en la realidad educativa a partir de correlaciones de cifras, sin establecer las mediaciones entre ambas.

Bajo el supuesto de otorgar validez a los resultados, se apela a modalidades de análisis que bajo el ropaje de cientificidad de los discursos y métodos hegemónicos procuran trasladar la discusión respecto a la legitimidad de la interpretación de los resultados al plano de lo técnico, como asunto propio de los expertos, dejando al margen cualquier referencia a los supuestos de los métodos mismos y a los asuntos de contexto y proceso que explican la emergencia de cierto tipo de resultados, relacionado directamente con el instrumento y modelo analítico utilizado.

\section{A manera de conclusiones}

- La promesa de no considerar concluyente ninguno de los componentes previstos para hablar de la calidad de la educación superior, y de buscar coherencia entre ellos, no tiene antecedentes en otras iniciativas gubernamentales de evaluación de la calidad, donde se ha mostrado más bien lo contrario.

- La precariedad de los procesos e instrumentos evaluativos en torno a los ECAES no permite hablar de los resultados de manera concluyente. Otra mirada frente al proceso produciría informa- ción que explicaría el instrumento logrado y sus resultados; al no reconocer estos aspectos 0 tomarlos como accesorios se evidencia una postura frente a los procesos evaluativos.

- Frente a los objetos de estudio, la evaluación y el concepto de calidad se pueden problematizar desde diversas posturas, con sus referentes conceptuales, epistemológicos y lógicos específicos. La reflexión sobre este asunto no sólo es indispensable para mantener la coherencia entre enfoques, modelos, métodos, técnicas e instrumentos, sino para comprender el logro de un instrumento, los resultados que produce y los efectos que sobre los evaluados se derivarían.

- Las pruebas de "lápiz y papel", para ser desarrolladas en un tiempo determinado,

- buscan establecer un aprendizaje concebido de manera simple, sin múltiples determinaciones;

- no dan cuenta de los aprendizajes procedimentales, saberes de tipo práctico que buscan solucionar problemas reales, tal como busca la formación universitaria;

- y subvaloran la mediación de la relación afectiva y de otras

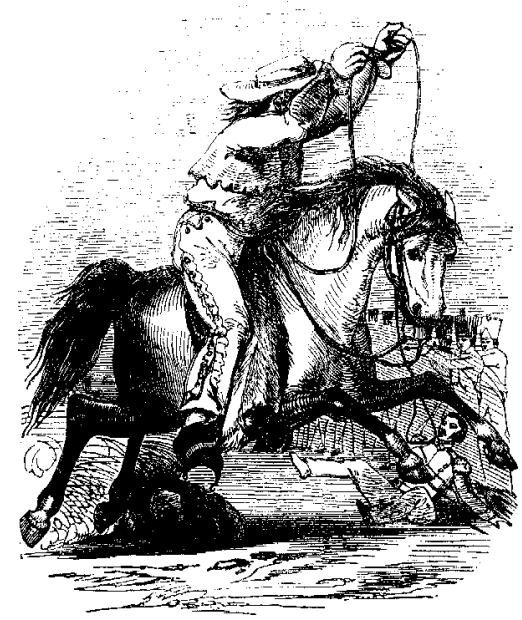

dimensiones que determinan la configuración de los aprendizajes.

- Desde varias disciplinas se ha mostrado que el desempeño en una prueba no revela la competencia, así se conciba ésta como algo universal o como algo situado.

- Las pruebas, iguales para todos en todas partes, poco indagan la determinación histórica, social, cultural y contextual de los aprendizajes.

- Según la psicología, de un lado, las pruebas condicionan en gran medida lo que buscan y entonces es necesario dar todo un rodeo metodológico para decir algo sobre el objeto; y, de otro lado, la dimensión cognitiva (la que busca la prueba) no es necesariamente la más importante en el ser humano, en todos las situaciones.

- Al sobredimensionar aspectos mejor desarrollados en algunos sujetos (con mayor acceso a herramientas culturales), y al olvidar el papel que dimensiones internas y externas cumplen en la apropiación de un saber, las pruebas terminarán siendo segregativas.

- El DNP y el MEN caracterizan la educación superior de manera que resulte "natural" la idea de los ECAES, de la reducción de financiamiento para la universidad pública, de una política de incentivos a la demanda; sin embargo, el Estado no se involucra en ese situación que denuncia, no reconoce su papel en la configuración del estado actual de las cosas.

- El uso de los resultados parece tener que ver más con la instrumentación de la prueba (diseño, modelo de análisis) que con la justificación o los propósitos desde los cuales las profesiones 
creen hacerse cargo de las pruebas.

- Los bajos resultados pueden legitimar acciones contra las instituciones, a nombre de la "calidad" de la educación superior. Si el acceso a determinadas instituciones queda en relación con las capacidades individuales, y no con las posibilidades materiales, las pruebas serían un mecanismo más de exclusión.

- La utilización de ciertas herramientas estadísticas no se ajusta a la naturaleza del objeto de interés, en este caso la educación; ello da cuenta de una cierta mirada frente al asunto, a partir de la cual se pueden legitimar acciones, y utilizar la técnica al arreglo de intencionalidades; bajo el recurso de apelar a criterios técnicos en los cuales los resultados son productos asépticos libre de cualquier interpretación errónea.

- Dado que ya emergen otras propuestas (como el observatorio al mercado laboral del MEN) que se articulan a la estrategia de evaluación ECAES y que pueden profundizar y reforzar las lógicas bajo las cuales opera y surte efecto dicha estrategia (según lo evidenciado en el texto: el fortalecimiento del modelo de mercado en la educación superior), valdría la pena que las instituciones de educación superior, comenzaran un proceso de discusión y construcción alterna acerca de los procesos evaluativos de la calidad de la formación profesional en Colombia. Se trataría de construcciones bajo otros supuestos epistemológicos y políticos que, en lugar de favorecer los mecanismos para que las instituciones y sujetos se incorporen a un modelo de funcionamiento que opera en su contra (en tanto surte efectos en las instituciones y sujetos a pesar del contexto mismo), permitiesen el reconocimiento de las condiciones a partir de las cuales se desarrollan los procesos formativos, en función de la transformación de los contextos desde una visión construida colectivamente.

- La evaluación de la calidad de los procesos formativos de los profesionales de la salud, de acuerdo con las condiciones de salud expresadas por los individuos y colectivos en Colombia, podría determinarse a partir de procesos evaluativos que den cuenta de la transformación positiva sobre las condiciones de vida, sobre el impacto en los fenómenos de salud-enfermedad, el impacto en la reducción de la mortalidad, en la producción de conocimiento pertinente para la resolución de las necesidades del país, lo que de paso permitiría a las universidades repensarse constantemente en procura de aportar en las construcción de posibilidades para el desarrollo y no limitarse a responder a las exigencias del mercado.

\section{Bibliografia}

Análisis de Rasch para todos, Una guía simplificada para evaluadores educativos. México, Ceneval, 1999.

Asociación Colombiana de Universidades. Consejo Nacional de Rectores. Documento a presidencia, octubre de 2003.

Ausubel -Novak- Hanesian. Psicología Educativa: Un punto de vista cognoscitivo. $2^{\circ}$, México, Trillas, 1983.

BarRantes, Esteban. "Política social, evaluación educativa y competencias básicas. Una mirada desde las políticas internacionales". En: El concepto de competencia I. Una mirada interdisciplinar. Bogotá, Sociedad Colombiana de Pedagogía, 2002.
Bustamante, Guillermo y Díaz, Luis Guillermo. Factores asociables al desempeño de los estudiantes. Bogotá, Universidad Nacional, 2003.

Ministerio de Educación Nacional. El Futuro de la Educación Superior. Plan Estratégico de Educación: un punto de partida. Enero 2002. (Este dato fue presentado en la esta reunión técnica, y lo sacamos de las presentaciones publicadas en la pagina web del ASCUN, consultado 13 mayo 2003).

Departamento Nacional de Planeación. 1994. En: Bustamente y Día: Factores asociables al desempeño de los estudiantes. Bogotá, Universidad Nacional, 2003. (esto está en la página 81).

Díaz, Mario. ¿Es posible evaluar objetivamente? Una aproximación crítica. En:. Evaluación escolar: ¿resultados o procesos? Bogotá, Magisterio 1996.

MARíN, Luis Fernando. «Competencias: "saber hacer", ¿en cuál contexto?». En: El concepto de competencia II. Una mirada interdisciplinar. Bogotá, Sociedad Colombiana de Pedagogía, 2002.

Nieva, BC. Jácome, S. Cendales, L. "Representaciones sociales del proceso salud enfermedad en poblaciones urbano marginales y su relación con los discursos y las prácticas institucionales". Revista Federación Odontológica Colombiana. Vol. 56 No. 194, septiembre - diciembre 1998.

PÁramo, Pablo. Nuestros vínculos con los animales. Bogotá, UPN, 1999.

SIMIAND, F. "El estadístico debe saber lo que hace": En: El oficio del sociólogo, de Pierre Bourdieu. México, Siglo XXI, 1988.

ToRrado, María Cristina. Hacia una cultura de la evaluación para el siglo XXI. Taller sobre competencias básicas. Edición especial. Bogotá, Universidad Nacional, 2000.

WolfF, W. Introducción a la psicología. México, Fondo de Cultura Económico, 1947.

ZuBIRÍA, M. "Del cociente intelectual a las inteligencias múltiples". Disponible en la página web del ICFES-Publicaciones fomento, consultado el 28 mayo de 2003). 